\title{
MICROSCOPIC IRREVERSIBILITY
}

\author{
KARL GUSTAFSON
}

Received 16 October 2003

First, I will recount the substance of several important conversations I had with Ilya Prigogine over the years. There is no doubt in my mind that Professor Prigogine firmly believed in the underlying stochasticity of the universe. Second, I will summarize my curiosity about the principle of detailed balance. In my opinion, so far it has always been put in by hand. Third, I will advance my own theory of microscopic irreversibility, that is, irreversibility at the quantum level. This involves new concepts of probability preference and probability violation. A manifestation of this irreversibility may be seen during emission of bound-free photons during field-matter interactions. Entropy increase is tied to radiation increase, and the notion of heat bath becomes light bath.

\section{Ilya Prigogine}

Professor Ilya Prigogine, Nobel Laureat 1977 (Chemistry), was one of the great visionaries of our time. I have written a personal remembrance elsewhere [19] and I do not want to repeat myself here. In this section, it is my intent to recount selected key conversations and discussions I had with him, especially as they pertain to certain longstanding fundamental issues in physics.

My association, and indeed, eventual friendship with Professor Prigogine began from a mathematical clarification we $[9,21,22]$ provided him concerning irreversibility of dynamical systems. As a result of this, Professor Prigogine came to Boulder to visit us and also gave the Edward Condon Memorial Lecture for the Department of Chemistry, April 16, 1979, and the annual Stanislav Ulam Invited Lecture for the Department of Mathematics, April 17, 1979. I hosted his visit, my wife and I had a small private reception at our home, and the chemists had huge receptions for him. I mention this because it set up the unusual situation whereby an outsider, a pure mathematician (me), found himself thrust into an open and friendly scientific dialogue with one of the world's great thinkers. I was not a threat in any political or scientific way because I was not engaged or employed within any of the competitive scientific arenas in which the great issues would embroil Professor Prigogine and the other noted physicists and chemists of his era. Also I was not a member of his Brussels-Austin team, even though I often visited the Solvay Institute 
in Brussels during the next 20 years. So, from his side, I was an honest sounding board. Also he liked to get the mathematician's reaction. From my side, it provided a wonderful learning experience as I tried to respond to his conversations and convictions.

In May 1985 Professor Prigogine called me from Texas: could I immediately rendezvous him in New York, to fly to Brussels overnight, to go to the aid of a colleague who had fallen ill? During the next week in Brussels we had many dinners and discussions together. Prigogine made it clear that he was seeking "irreversibility at the microscopic level," in other words, if possible, an extension of thermodynamics all the way down to the level of quantum mechanics. In my private notes, (Colorado, 1985), written shortly thereafter, I created my own scenario as follows. Accepting the Schrödinger equation as correct enough by itself, that is, without thermodynamic considerations, then an initial (probabilities) state $u(0)$ is unitarily (reversibly) evolved but the spreading of the wave (probabilities) packet means that, for a particular region of configuration space, the probability of the particle (e.g., electron) being there decreases. This corresponds to the (eventual) decay of the electron toward lower (atomic) energy states. Thus some kinetic energy must have been transferred elsewhere. Where? I speculated that it had gone into a "more disordered" state manifested by the more uniformly spread-out wave packet. This view presupposes an initial wave packet prepared with some central tendency, like a Gaussian for example. Then the evolving wave packet $u(t)$ is pictured as a Gaussian with larger variance. That larger variance corresponds to a higher "volatility" of the electron position (probability) and that volatility increase cannot be reversed (such would require outside energy to be put in to effect a transition to a higher energy level). Thus the increasing loss of central (probability) tendency is a second law. This view, which I am sure is not novel, is also pretty simplified: one could bring in to it other features such as photon loss due to downward transitions; one could try to interpret the probability spreading as heat transfer, and the like.

But Professor Prigogine was seeking something more objective. In our conversations, I had pressed him for what he was really searching for, and he said: "something like a Schrödinger equation for thermodynamics." By this he did not mean really a Schrödinger equation, and of course his work had extensively used master equations and other statistical transition equations. I believe that he was hoping for a fundamental, almost deterministic partial differential equation which could describe thermodynamics at the quantum level. He wanted [39] chance to play a role only at or near bifurcation points. Between such critical junctures, perhaps there was a fluctuation-driven but nice fundamental evolution equation to be discovered.

Professor Prigogine visited me again in 1992 in Boulder, giving a large public lecture. He also requested one "totally free day to just visit," so we went up to my small mountain cabin for the day. During all our conversations in this visit, Professor Prigogine made clear to me how much he opposed and had always opposed the Einstein view. Prigogine emphatically believed in the underlying stochasticity of the universe. On the other hand, there is no doubt in my mind that Prigogine shared with Einstein the hope that mathematics, with its intrinsic beauty and consistency, could provide conceptual breakthroughs. For this reason, he was always searching for some mathematics to explain the order of time. Relying on coauthors who were more mathematical than him, 
sometimes he believed that a mathematical, intrinsic ordering of time had been achieved. Being more mathematical than his coauthors, I could see where, in my opinion, time order had been put in by the scientist, through certain, sometimes subtle, choices in the mathematics. Usually I kept my opinion to myself but sometimes he confronted me directly and I could not avoid telling him my view. These were the only times he became angry with me even though in polite terms. You will find my countervailing views fully expressed in [13].

Often we talked past each other on such issues, nonetheless communicating. An example is the following. I sometimes made a few cryptic notes after our conversations, usually not. But one time, after a discussion in 1992, I wrote down quickly in my private notes (Brussels, 1992) as verbatim as possible our dialogue. Here it is. We had agreed that quantum mechanics was the Schrödinger equation and the spreading of the wave packet.

Karl Gustafson: But where does the information go?

Ilya Prigogine: Into entropy increase. That is the second law. And quantum mechanics does not preserve information.

Karl Gustafson: I mean, like in fluid turbulence. One wants a transition to higher frequencies but so that those oscillations keep nontrivial amplitudes.

Ilya Prigogine: That is what we lose in the asymptotic limit. That is what the restriction to test functions does to us. So the test functions become part of the description.

Karl Gustafson: But the test functions spaces are not unique.

Ilya Prigogine: We need the physical ones.

Karl Gustafson: Smaller test function spaces mean larger dual spaces hence more functionals to represent measurements.

Ilya Prigogine: Quantum mechanics must have the property of coherence.

Karl Gustafson: The larger the dual space, the more eigenfunctionals, the less coherence.

Ilya Prigogine: The Schrödinger equation preserves trajectories so cannot be irreversible. The test function space dual does not. It is like radiation.

Karl Gustafson: Like the limiting absorption principle, or even the improper eigenfunctions in the Dirichlet Problem.

Ilya Prigogine: We choose our own nonlocalizing, the test functions, the coherence.

Karl Gustafson: I prefer to think nature should do that, more like smoothing, regularizing.

Ilya Prigogine: At least that is our human picture.

This conversation is interesting to me because in it eventually Professor Prigogine himself expresses my reservations (human modeler set time order). However, when I went on to my principle of regularization [13], he misinterpreted my view (I want nature to do it all, just as he wanted) and made very clear to me his opposition to the idea that "physics depends on the human observer." How could that be, that my approximations to nature could affect nature, another's approximations would lead perhaps to a different result, and the like, I cannot remember exactly. Notice that I used the word "approximation" and not the word "observation" here. We were both using the word approximation, and he misinterpreted my view. But he was adamantly against the notion that fundamental physics must be subjective. He wanted something more objective, even if stochastic. 
Let me carefully elaborate this point, as it bears important issues related to mind and matter. Currently, and indeed for a long time now, there are many debates about whether the "Bohr" or the "Einstein" view of quantum physics is correct. It seems to me not fair, not even correct, to force one to absolutely choose between one or the other of these countervailing positions. However, to a first-bit precision, you could put Professor Prigogine in the first camp, and me in the second camp. But when you add a second bit of precision, both Prigogine and I would agree: we would be in the "stochastic" camp, not in the "classical" camp. Further details on my opinions may be found in [17].

\section{Detailed balance}

This brings me to the second point I would like to elaborate, which I will call the joint issue of detailed balance and exchange of stabilities. Even before I met Professor Prigogine, I had become interested [10] in the "principle of exchange of stabilities" as we studied it in fluid dynamics. From his book [8] I became acquainted with the "principle of detailed balance." Exchange of stabilities also plays a frequent role in the important book [8]. It became clear to me rather quickly, however, that Professor Prigogine looked at bifurcation theory quite differently than did we mathematicians who had been studying it. To us, the case was that we were given some nonlinear differential equation, for example, the Navier-Stokes equations of fluid dynamics [10, 15], and we wanted to rigorously justify, for example, the "branching" of a steady solution into a periodic solution as a critical physical parameter increased through a bifurcation point. To Professor Prigogine, the situation was less continuous; what was a periodic solution to be achieved by us was to him a "far from equilibrium" solution, and for him it occurred $[8,39]$ due to the buildup of background stochastic fluctuations which eventually forced a transition.

There is too much to discuss here, but let me state that I think the issues surrounding and joining detailed balance and exchange of stabilities within thermodynamics and statistical mechanics are far from resolved. And one finds (see, e.g., [23]) instances of a detailed balance concept in quantum mechanics, for example, an atom of several levels in resonance with a light field or radiation field. From the first time I saw the "principle of detailed balance," I had become curious about it. Recently I was by chance in Trondheim lecturing in May 2002 and there by chance I looked closely at The Collected Works of Lars Onsager [34]. There I found the answer to my curiosity: Onsager had put in the "detailed balance" by hand, saying something to the effect that things must be reversible, like Newtonian dynamics, at the molecular level. Specifically, in his Nobel lecture, Onsager says: "for the relaxation effect I could depend on Newton's principle of action and reaction" [34, page 52]. He also argued that the principle of detailed balancing was thermodynamically equivalent to a principle of least dissipation (of energy).

If you look carefully at their book [8, pages 32 and 117] you will see that Prigogine and Glansdorff also put in a detailed balance principle by hand. To my mind, this leaves deeper issues unresolved, but to those gifted few who just "see it," it is clear. Intuition is so strong that there can be no doubt. As I said above, Prigogine was always looking for something deeper in thermodynamics, although it seems that he did not relate his continuing search to detailed balance, which he just accepted. However, perhaps because of my earlier experiences in hydrodynamics where it is not easy to actually rigorously 
prove a principle of exchange of stabilities, especially when one stays in the nonlinear equation regime, I found detailed balance a property to be proved, not assumed. And if one cannot prove something mathematically, often there is a deeper secret to be unlocked.

Therefore, in this section, I would like to discuss for the first time certain aspects of detailed balance. I have not presented any such discussion elsewhere. I am especially interested in detailed balance eventually in the context of quantum mechanics, specifically as to how its statics and dynamics may be synonymous with that of a hidden second law. However, we first recall that role of detailed balance in statistical physics. See $[8,31,34,35]$ and the citations therein for more details.

Onsager's original two (1931) papers [34] stressed that one should take matrix elements $L_{i j}=L_{j i}$ so that the antisymmetric part of a quadratic form generated by $L$ vanishes so that the form would be symmetric positive semidefinite. These "reciprocity relations," I would call them assumptions rather than relations, were motivated as mentioned above by a tacit ansatz of Newton's principle of action and reaction. There was also a lot of approximate experimental evidence. Also "for all the complications of hydrodynamics a "principle of least dissipation" derived by Helmholtz assured the symmetry," [34, page 52]. Also "in the state of equilibrium, the reaction $1 \rightarrow 2$ would occur just as often as $2 \rightarrow 1, \ldots$, " [34, page 52]. Here the $L_{i j}$ produce the ionic fluxes $J_{i}=\sum_{j} L_{i j} k_{i}$ from the $k_{i}$ forces on the ion. From these various assumptions (linearity, symmetry, and positivity), one may assure a nonnegative entropy production throughout the system.

These themes are carried forward throughout the Prigogine-Glansdorff book [8]. As summarized [8, page 42], they wish to establish "Helmholtz's theorem according to which laminar flow corresponds to a minimum of energy dissipation. Under isothermal conditions, this is equivalent to minimum entropy production." However, from the mathematician's view, nothing has been proven. The mathematical analyses are all formal and the modelling, while excellent, all follows from ansatz or physical reasoning. Shortly after my discovery of Onsager's collected works [34] in Norway in 2002, I happened to spend a week at the Solvay Institute in Brussels and there I again checked my reading of Prigogine's book [8] and I am quite sure of my opinion. My comments apply as well to the various considerations involving the principle of exchange of stabilities in [8]. For example, "every time the antisymmetric contribution II in the evolution criterion may be eliminated by a suitable choice of the weighting functions, the principle of the exchange of stabilities is valid," [8, page 168]. Mathematically, this amounts to removing any possibility of a nontrivial imaginary part of the energy or entropy quadratic form being treated at a bifurcation point. Thus there is no dissipation there. Thus no energy is lost. Thus stability is exchanged at no cost. This is the thinking.

When I first encountered the principle of exchange of stabilities in hydrodynamics, I found that everyone referred to Chandrasekhar's book [6] as having proved it. But a close reading of [6] shows it has not been proved. Chandrasekhar, like Onsager and Prigogine, has linearized the dynamics under discussion. Or one may say that, based upon abundant approximate experimental evidence, what they wish to claim is approximately true. This distinguishing of "assumption" from "derivation" is critical to a mathematician. Even excellent mathematicians can sometimes push forward and lose sight of the difference. For example, when the book [32] came out, I carefully examined it and found that although 
it constitutes an essentially correct and useful mathematical treatment of bifurcation theory for fluid dynamics, when it comes to the application to the actual full Navier-Stokes equations for fluids, the hypotheses needed for proving the Hopf bifurcation have not been shown to be present.

As Mazur [33] recounts in [34], Casimir and Bridgman, two excellent scientists, had discomfort with Onsager's claims. Eventually, it becomes clear that the reciprocal relations are only approximately valid (see [33]). As I point out in [15, page 13, see also Section 1.8], "there is a great conceptual difference between linear and so-called nonlinear eigenvalue problems."

We consider next detailed balance as it is treated in quantum mechanics. In the classic book [23], in a treatment of the scattering matrix, detailed balance is first regarded as the neglecting of the damping terms. Again, this amounts to an ansatz of no dissipation, or at least, the neglecting of such. However it gets more interesting [23, pages 163-174] where damping is treated. For an atom in a discrete excited level, there will be a finite transition probability per second into lower energy states, accompanied by photon emissions. Such states have a finite level "width" $\Gamma=\mathscr{R}(\Gamma)+i \mathscr{I}(\Gamma)$ whose real part $\mathscr{R}(\Gamma)$ is the total transition probability per second from that state into all other states, whereas $\mathscr{S}(\Gamma)$ is a change of the energy of the state itself. Thus $\mathscr{R}(\Gamma)$ will implement the energy transfers to other states and $\mathscr{I}(\Gamma)$ would displace the spectral line of the "transmitting" state. To avoid this complication of having to deal with the imaginary quantity $\mathscr{I}(\Gamma)$, it is assumed [23, page 168] that one may instead use a representation wherein self-energies are already included in the energy values $E_{n}$, so that one may assume that $\mathscr{(}(\Gamma)=0$. We may editorially interject here how overly convenient, just as in our discussion of the principle of exchange of stabilities above, it is to assert a "no cost," in this case, no line displacement energy transfer. However, Heitler [23, pages 172-174] carefully then considers the line displacement "self-energy." A representation (energies become those of a difference of Hamiltonians) is produced. The "disconcerting," "asymmetry between initial and final states has disappeared," but Heitler points out that, strictly speaking, that is not the case, due to the dependence of $\Gamma$ on $E$. So the problem has not gone away. Later [23, page 337] he shows that to remove this asymmetry, one must permit a change of mass in the base Hamiltonian as a single electron drops to the ground state. Then $\mathscr{I} \Gamma\left(E_{n}\right)$ is very small (but not zero), but the self-energies of the bound states "do not merely reduce to a mass correction but are finite and observable, after mass and charge renormalization." We editorially interject that the plot to get a "detailed balance" is getting more complicated. A way to approximately calculate the exact level shift is described. It is assumed that an electron can emit or absorb a photon without changing its state (to this author, this is a hidden detailed balance ansatz; a "no cost" entity change). Eventually one must subtract off self-mass and self-charge. The considerations [23] continue on and on. One remains enmeshed in the divergencies of QED.

Heitler later [23, appendix, page 412] returns to detailed balance per se. He recalls that in statistics, as we described above, the concept is used as a sufficient condition to guarantee increase of entropy and the attainment of statistical equilibrium. However, for quantum mechanics, he needs to further assume time-reversal invariance and space-reflection invariance. This results in a principle of detailed balance but at a cost of reversing the 
spin directions. Quoting [23, page 414], “in general one cannot expect the principle of detailed balance to hold when angular momenta, polarizations, etc., are involved, except to a first approximation."

In discussing time-reversal invariance in multichannel scattering, Taylor [42, page 354] carefully distinguishes the principle of detailed balance, the reciprocity theorem, and the principle of microreversibility. Amrein et al. [1, page 292] interpret quantum detail balance in terms of the scattering optical theorem (when $S$-matrix is unitary) as meaning the total cross section is identical to summing the differential cross sections over all initial directions. For a more modern view of the treatment of threshold displacements via the renormalization group, see [3].

It is not the purpose of this paper to provide a further comprehensive survey of detailed balance. In thermodynamics one wants a symmetric positive definite linear system upon which to do variational calculus and to get that Onsager assumed, that is, put in by hand, a Newtonian microdynamics. In statistical mechanics and linearized fluid dynamics, one assumes, that is, puts in by hand, at bifurcation points that the dynamics acts as in Hamiltonian dynamics, that is, is governed only by real energies and linearized laws and not by any imaginary entities. In quantum mechanics, one assumes that the probability of spontaneous emission of a photon from an atom is the same as the probability of such emission as induced by the field. Thus we may say that it is always to be as in Newton's second law. In the microdynamics, it is to be "action = reaction," an exact energy exchange, the same probabilities of coming and going.

\section{Quantum microirreversibility}

I put forth in [12, pages 68-76], see also [13, pages 501-506], an idea which could become a foundational basis of microirreversibility in quantum mechanics. Strangely enough, this idea came to me in 1990 while I was preoccupied with research in optoelectronic artificial neural networks [11]. Here I would like to summarize what I said in $[12,13]$ and go further. For a collateral discussion of the importance of these ideas within the science of neural networks, see $[14,16]$.

The microdynamics of neurobiology has established experimentally and phenomenologically $[24,25,26,27,29]$ that a sigmoid curve (with the appropriate parameters and units) describes the change in chemistry that takes place during a neural synapse. Working in artificial neural networks, one also employs such a sigmoid function, $F(x)=(1+$ $\left.e^{-\beta(x-\theta)}\right)^{-1}$, to implement learning. Here $\theta$ is threshold; $\beta$ is gain. Such curves also occur in statistics where they are often called logistic distribution functions: poor man's approximations to normal distributions. I noticed [11] that chaos sometimes occurred in our digital simulations when the gain $\beta$ exceeded 4 . This is another story, see $[14,16]$, but I also realized a fact that I had always overlooked within the science of statistics. Let $f(x)$ be any probability density function and $F(x)$ its corresponding probability distribution function. Moreover, let $\{F(x)\}$ more generally denote the class of all potential distribution functions monotonically increasing from 0 at $-\infty$ to 1 at $+\infty$. Then one may state a lemma: the class $\{f\}$ of probability density functions and the class $\{F\}$ are not one-toone. There are more of the latter. For logistic probability distributions, when $0<\beta \leqq 4$, 
the relationship is one-to-one. But when $\beta>4$ in $F$, its derivative $f$ exceeds the value 1 at the mean threshold value $\theta$. I call this probability violation $[12,13]$.

Consider now when we are speaking of quantum mechanics. In the usual complex Hilbert space formulation, position and momentum are the basic observables, correspond to selfadjoint operators, evolve as unitary exponentials of those infinitesimal generators, and have spectral measures deducible from those operators. By the Stone-von Neumann theorem, these operators combine to form a system of imprimitivity from which it can be shown that every irreducible representation is isomorphic to the Schrödinger representation in $\mathscr{L}^{2}\left(\mathbb{R}^{n}\right)$. For the position operator for example, its spectral measure $E(I)$ measures the probability of finding the object in the interval $I$, and in the Schrödinger representation this may be computed by $(E(I) \phi)(x)=\chi_{I}(x) \phi(x)$ for a given state vector $\phi(x)$, where $\chi$ here denotes the indicator function of the interval. Generally, for selfadjoint operator observables $A=\int \lambda d E_{\lambda}$, we have the state $\phi$ distributions $F_{\phi}(a, b]=\int_{a}^{b} d\left\langle E_{\lambda} \phi, \phi\right\rangle$. In terms of the lemma I stated above, I wondered (1990) what would happen if a quantum spectral density function $f_{\phi}(x)=d\left\langle E_{\lambda} \phi, \phi\right\rangle / d x$ for some observable $A$ and some state $\phi$ was forced by an event to exceed its allowed steepness and thereby incurred what I call probability violation.

To save space I now refer to $[12,13]$ for further details concerning the following. Briefly, I formulated a principle of probability preference: nature prefers to preserve probability densities. When the probability density amplitude exceeds one, information is lost. This may signal an irrevocable irreversibility at the fundamental level. Using the sigmoid (logistic) probability distribution as an example, I noted that the gain $\beta$ acts as a width parameter in the probability density which behaves very much like the Breit-Wigner formula. When probability preference is violated due to too much gain, the radiation energies have exceeded some fundamental informational limit. That would constitute irreversibility.

Further $[12,13]$, when the probability density $f_{\phi}(x)$ is regarded as a spectral concentration over the continuous spectrum due to a scattering event, we may interpret such as a photoeffect in which an incoming photon is absorbed, creating an energy transfer from a high energy bound state to an increased spectral concentration at some threshold $\theta$ within the continuous spectrum. Inasmuch as the line width $\Gamma$ may be interpreted [41] as the transition probability per unit time for spontaneous emission, any measurement of the high energy bound state must be shorter than the $\beta^{-1}$ lifetime of that state. By the uncertainty principle, the measurement accuracy cannot exceed $\beta \hbar$. Thus the measurement causes a shortened lifetime of the high energy bound state and an emitted line broadening of magnitude $\beta$. Moreover, when the gain $\beta \cong \Gamma$ exceeds the allowed spectral concentration steepness, absorption of the photon by the high energy continuum states near the threshold $\theta$ and conservation of energy cause a probability violation, a loss of quantum probability, which I would regard as quantum microirreversibility. See the schematics [12, Figure II-1], [13, Figure 14].

If we take a semiclassical treatment of quantum radiation, that is, we treat the electromagnetic field classically and the particles interacting with it quantum mechanically, then Schiff [41, Chapter 11] gives an account of the influence of the field on the particles, but not conversely. Notice that my scheme above attempts to do the converse, that is, it 
tries to give an account of the influence from particle to field. By a dipole approximation assumption, $e^{i k \cdot r} \cong 1$, one arrives (see [41, page 405]) at Einstein's formula [7, 38] for the transition probability per unit time for absorption and induced emission, namely, $\left(4 \pi^{2} e^{2} / 3 \hbar^{2} c\right) I\left(\omega_{k n}\right)|\langle k|r| n\rangle|^{2}$. Recall that here $I$ is the intensity of the impinging radiation. I would like to note here for further comment below that in this semiclassical view one has tacitly accepted a kind of linearization, that is, what I would call a Hooke's Law: emission probability is proportional to the intensity of the radiation. Continuing to get a quantum theory, Schiff [41, pages 413-414] replaces the dipole assumption by a quantum current density for the energy related in a $k$ to $n$ upper to lower (bound) state transition. This is put in by hand but Schiff is careful to admit that. This leads to the quantum Einstein formula for the transition probability per unit time for spontaneous emission, namely, $\left(4 e^{2} \omega_{k n}^{3} / 3 \hbar c^{3}\right)|\langle k|r| n\rangle|^{2}$. Moreover, one obtains [41, equation (45.23)] Planck's formula $[7,38]$ for the spectral distribution of thermal radiation in a cavity, namely, the energy density is $I(\omega) \Delta \omega / c=\left(\hbar \omega^{3} \Delta \omega / \pi^{2} c^{3}\right)\left(e^{\hbar \omega / \kappa T}-1\right)^{-1}$. However, to obtain this, one uses Boltzmann's statistical mechanics equilibrium ratio $e^{-\left(E_{k}-E_{n}\right) / \kappa T}$ of the number of particles in upper energy state to that in lower energy state. Thus Planck's formula is a result of a statistical mechanics detailed balance assumption. The Einstein quantum probabilities for absorption of a light quantum, namely, $\omega_{\text {emission }} / \omega_{\text {absorption }}=\left(n_{\nu}+1\right) / n_{\nu}$, are also a semiclassical detailed balance. Also I note that "the probability of absorption is proportional to the intensity of the incident light beam as is to be expected," [23, page 179]. Thus Heitler [23] (tacitly) accepts the "Hooke's linearization" assumption I referred to above.

My point is that we continue to find under close examination that, just as Onsager did for his desired chemical rate equation detailed balance, even Planck's statistical mechanics detailed balance and Einstein's quantum mechanical detailed balance are based on tacit, undeclared assumptions that the microworld will follow a Newton, linearized approximate dynamics. In each case this is put in by hand, albeit well buttressed by strong experimental evidence or profound physical intuition.

Under my theory for a quantum microirreversibility (to some extent, put in by hand), an event which produces too much spectral concentration steepness among the "free" states can be interpreted as a violation of probability and consequent loss of information and therefore constitutes a fundamental quantum mechanical microirreversibility. Macroreversible quantum mechanics and my principle of probability preference would assert that nature will not want to let that happen. Suppose one or the other is true. The former would amount to a second law, in which the classical concept of entropy production into a heat bath is replaced by a notion of quantum radiation increase into a light bath. The latter would say the kicked up and to be locally rejected energy would have to go, say, to another atom, or to complex resonance phenomena, or elsewhere, but still in some form retaining its "informational," that is, probabilistic, content. Or both could be true; there could be a perpetual small probability loss going on all the time in the "flickerings" of atoms in their fields, but it would be premature to engage such speculation. Also I do not want to speculate on the nature of the light bath, for example, one should keep in mind that rather than being some exotic entity like dark matter, it should be what "lost" probability would be. 
In their treatment of the photoeffect, Bethe and Salpeter [5, page 295] treat "boundfree" radiative transitions. One finds that $\omega_{W}=N \circ \omega_{W}$ and $\tau_{W}=N \sigma_{W}$ for the probabilities per second, respectively, that one atom is excited to energy $W$ and that one photon is absorbed in a $1 \mathrm{~cm}$ path length. Here $N$ 。 is the number of photons incident upon the atom, $N$ is the number of atoms per $\mathrm{cm}^{3}$ in the state being struck by the radiation, and $\sigma_{W}$ is the cross section for the quantum numbers being considered. Bethe and Salpeter [5] also consider an alternate, scattering wave function-based paradigm for calculating the absorption probabilities. Then it is admitted that, either way, exact expressions for the photoeffect cross sections are not available, so one must go to approximations, and a number of those are considered (screening, Hartree approximations, dipole approximation, and Born approximation; see [5, pages 297-320]). Then the Bremsstrahlung (freeto-free transitions) is considered. My ansatz and description above of potential probability violation caused by over-steepening of the spectral distribution functions over the continuous spectrum and its possible implications toward quantum microirreversibility are equally relevant for the free-to-free transitions.

\section{Concluding discussion}

When I first started taking physics courses in my youth, I was uncomfortable with how the teachers "isolated systems" in order to then analyze them. I was always asking myself, how can they ignore what is happening at the boundary, or in the next adjoining system? Essentially, I already had an intuition that everything is connected, nothing is exactly reversible; in other words in technical terms I learned later, there are no exact Hamiltonian systems in nature. Looking back now, I see that my intuition was naturally compatible with that of Professor Prigogine. Also I was well versed in the mathematical theory of dissipative semigroup evolutions, a tool which fits naturally into his investigations at that time.

On the other hand, Prigogine's beliefs that entropy was the fundamental quantity of statistical physics, and that unstable systems a la Poincare were the key to understanding the fundamental dynamics, did not convince me. Entropy is too macroscopic; to me it is an emergent entity, and moreover, for example in numerical schemes for first-order hyperbolic systems of partial differential equations (e.g., gas dynamics, see [15, Sixth Pause]), one has several entropy inequalities from which to choose to stabilize the algorithms. As for unstable systems, Poincaré recurrences, homoclinic points, and the like, while seeing them fascinating, I could never shake the feeling that physicists were just jumping on the bandwagon of the new mathematical discoveries in the qualitative theory of systems of nonlinear ordinary differential equations in three- and higher-space dimensions. Moreover, one does not need chaos to have instability. Hadamard's old linear partial differential equation non-well-posed initial value problems [15, page 134] will do. Furthermore, as I made clear in [12, 13], I see no physical magic and no time ordering in rigged Hilbert spaces.

More generally, perhaps a few (three) qualifying or related side remarks are in order here. First, even though my ideas for probability violation as a potential second law for quantum mechanics originated in 1990 during my work (see $[11,14,16,24,25,26,27$, 
29]) with neural networks, I am in no way supposing any connection in nature between the two. Specifically, I am not asserting, as Penrose $[36,37]$ does, that we must couple the human consciousness to quantum mechanics in order to understand both. That may or may not turn out to be the case, but my point here is that, in this paper, I am not dealing at all with all the facets of irreversibility having to do with quantum measurement theory, the loss of unitarity in time evolutions, and the like. Some comments about those issues may be found in $[13,17,20]$. In the latter, I provide a new Zeno alternative formulation whereby the unitarity dynamics of evolution may be maintained in the presence of measurement provided that one demands that one's "counters" or measuring observables have the potential to measure every state $\phi$ in the domain of the Hamiltonian which generates the evolution. I comment that this new result [20] has nothing directly to do with human consciousness or observer.

Second, I am not addressing in any way some of the grand issues such as universal wave function, causality, nonlocality, gravitation, and the like. A delightful discussion of such may be found in the recent book [40]. In [18] I do take the position that Bell's inequalities are really just aspects of the geometry of Hilbert space, and consequently, from which one has no right to draw any conclusions whatsoever about nonlocality.

Third, I have not speculated on what may be a deeper foundational (still one level finer ...) mathematical/physical basis of probability violation and probability preference in quantum mechanics. That is, I can relate these in the sense of statistics, that is, probability distributions, to the photoeffect, but I cannot tell you the underlying event algebra which would tell you when or which photon decides to jump to the continuum. In [2] we argue how probability can arise from underlying chaos, but I would not guess that any such chaos (in the usual sense) or Kolmogorov mixing, and the like, would be the reason here. Rather, it may lie in the mysteries of just what a field really is, or in the nature of the atomic radius and in the interaction zones between atoms.

Concerning detailed balance in quantum mechanics, Ballentine [4, page 362] notes that Einstein [7] was able to deduce some of the main features of spontaneous emission of radiation by an atom dropping to its ground state, well before most quantum theory was known. He then goes on to give the essentials of Einstein's argument. Einstein's basic assumption was that the radiation mechanism must preserve the statistical equilibrium among the excited states of the atom. Thus his detailed balance became "probability of induced emission = probability of absorption." But as I pointed out above when discussing all of the intricacies of renormalization and radiative corrections as treated in Heitler's book [23], there is the (tacit) deeper assumption that an electron can emit or absorb a photon without changing its state. I find this to be an example of the worries I incurred as described in the first sentence of this section. More to the point, the capture or release of a photon by an electron could well cost something, that is, it could be a small but everpresent quantum irreversibility. My intuition says there is some kind of mechanical work being done, figuratively some friction, some cost, in capturing a photon. Of course this intuition is based upon a semiclassical, scattering view of such dynamics.

I also just take note of the recent photonic crystal emission findings [30, 44] which appear to contravene Planck's [7,38] radiation law. Of course the tungsten-based photonic crystals described in $[30,44]$ and their remarkable funneling of energies to short 
wavelengths with emissions greater than that allowed by Planck's black-body limit are mesoscopic structures, but it makes one wonder why the detailed balances of quantum mechanics (which are statements about probabilities) need to conform to classical thermodynamics.

To conclude, my ansatz of probability violation and probability preference as a second law for quantum mechanics as described in this paper and $[12,13]$ has been formulated in terms of mathematical spectral theory. What specific physical quantum observables $A$ and perhaps functions $f(A)$ of them will have spectral families predisposed to be vulnerable to probability violation and hence to fundamental quantum microirreversibility? Spectral concentration is well known [28] in scattering theory and my theory should also be further investigated within that context. Most important physical experiments would be most valuable, but since we are speaking of probabilities rather than events, such experiments could be difficult to carry out.

To close on a philosophical note, we choose and ponder the nice quotation [43, page 65]: "chance is in truth only another name for ignorance of the causes."

\section{References}

[1] W. O. Amrein, J. M. Jauch, and K. B. Sinha, Scattering Theory in Quantum Mechanics, W. A. Benjamin, Massachusetts, 1977.

[2] I. Antoniou, Th. Christidis, and K. Gustafson, Probability from Chaos, to appear in Internat. J. Quantum Chemistry.

[3] V. Bach, J. Fröhlich, and I. M. Sigal, Mathematical theory of nonrelativistic matter and radiation, Lett. Math. Phys. 34 (1995), no. 3, 183-201.

[4] L. E. Ballentine, Quantum Mechanics, World Scientific Publishing, New Jersey, 1998.

[5] H. A. Bethe and E. E. Salpeter, Quantum Mechanics of One- and Two-Electron Atoms, SpringerVerlag, Berlin, 1957.

[6] S. Chandrasekhar, Hydrodynamic and Hydromagnetic Stability, The International Series of Monographs on Physics, Clarendon Press, Oxford, 1961.

[7] A. Einstein, Zur Quantentheorie der Strahlung, Phys. Z. 18 (1917), 121-128 (German).

[8] P. Glansdorff and I. Prigogine, Thermodynamic Theory of Structure, Stability, and Fluctuations, John Wiley \& Sons, New York, 1971.

[9] K. Goodrich, K. Gustafson, and B. Misra, On converse to Koopman's lemma, Phys. A 102 (1980), no. 2, 379-388.

[10] K. Gustafson, Asymptotic distribution of eigenvalues of product operators determining hydrodynamic stability, Amer. Math. Soc. Not. 23 (1976), A-682.

[11] - Reversibility in neural processing systems, Statistical Mechanics of Neural Networks (L. Garrido, ed.), Springer-Verlag, Berlin, 1990, pp. 269-285.

[12] Lectures on Computational Fluid Dynamics, Mathematical Physics, and Linear Algebra, World Scientific Publishing, New Jersey, 1997.

[13] _ Operator spectral states, Comput. Math. Appl. 34 (1997), no. 5-6, 467-508.

[14] Internal sigmoid dynamics in feedforward neural networks, Connection Science 10 (1998), 43-73.

[15] _ Introduction to Partial Differential Equations and Hilbert Space Methods, Dover Publications, New York, 1999.

[16] Chaos in discrete learning systems, Chaos Solitons Fractals 11 (2000), no. 1-3, 321327. 
[17]

[18]

[19]

[39] I. Prigogine and I. Stengers, Order out of Chaos, Bantam Books, Toronto, 1984.

[40] T. Rothman and G. Sudarshan, Doubt and Certainty, Perseus Books, Massachusetts, 1998.

[41] L. Schiff, Quantum Mechanics, McGraw-Hill, Tokyo, 1968.

[42] J. Taylor, Scattering Theory, John Wiley \& Sons, New York, 1972.

Chance (H. Atmanspacher and R. Bishop, eds.), Imprint Academic, Thorverton, 2002, pp. 115-148.

Conference on Physics (I. Antoniou, V. Sadovnichy, and H. Walter, eds.), World Scientific Publishing, New Jersey, 2003, pp. 534-554.

(2003), 9-13. Physics-2 (A. Khrennikov, ed.), Växjö University Press, Sweden, 2003, pp. 225-236. Soc. János Bolyai, vol. 35, North-Holland Publishing, Amsterdam, 1983, pp. 567-579.

hardt, eds.), Plenum Press, New York, 1981, pp. 203-210.

W. Heitler, The Quantum Theory of Radiation, 3rd ed., Oxford University Press, London, 1954.

A. Hodgkin and A. Huxley, The components of membrane conductance in the giant axon of Loligo, J. Physiol. 116 (1952), 473-496.

of Loligo, J. Physiol. 116 (1952), 449-472.

J. Physiol. 116 (1952), 497-506. excitation in nerve, J. Physiol. 117 (1952), 500-544.

T. Kato, Perturbation Theory for Linear Operators, 2nd ed., Springer-Verlag, New York, 1976.

S. Kuffler, J. Nicholls, and A. Martin, From Neuron to Brain, 2nd ed., Sinauer Associates, Massachusetts, 1984. near a photonic band edge, Applied Physics Letters 83 (2003), no. 4, 593-595. (2) 91 (1953), 1512-1515. matical Sciences, vol. 19, Springer-Verlag, New York, 1976.

P. Mazur, Onsager's reciprocal relations and thermodynamics of irreversible processes, The Collected Works of Lars Onsager. With Commentary, World Scientific Series in 20th Century Physics, vol. 17, World Scientific Publishing, New Jersey, 1996, pp. 61-70.

L. Onsager, The Collected Works of Lars Onsager. With Commentary, edited by P. C. Hemmer, H. Holden, and S. Kjelstrup Ratkje, World Scientific Series in 20th Century Physics, vol. 17, World Scientific Publishing, New Jersey, 1996. $1505-1512$.

R. Penrose, The Emperor's New Mind, Oxford University Press, New York, 1989. , Shadows of the mind, Oxford University Press, Oxford, 1994.

M. Planck, Vorlesungen Uber Die Theorie der Warmestrahlung, Verlag Johann Ambrosius Barth, Leipzig, 1923. 


\section{Microscopic irreversibility}

[43] G. Thomson, The Atom, Oxford University Press, Oxford, 1963.

[44] P. Weiss, Hot crystal, Science News 164 (2003), no. 14, 218-220.

Karl Gustafson: Department of Mathematics, University of Colorado, Boulder, CO 80309-0395, USA

E-mail address: gustafs@euclid.colorado.edu 


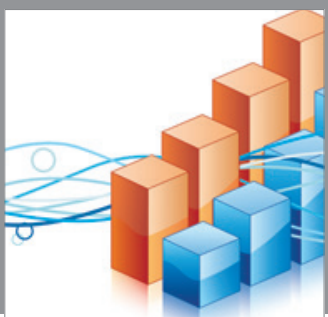

Advances in

Operations Research

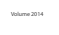

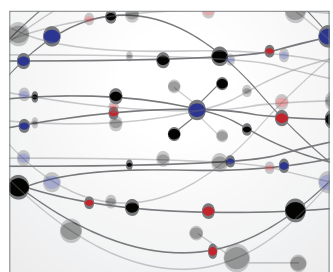

\section{The Scientific} World Journal
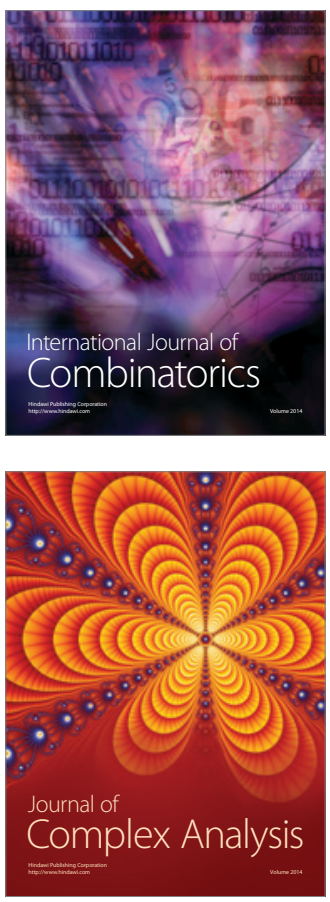

International Journal of

Mathematics and

Mathematical

Sciences
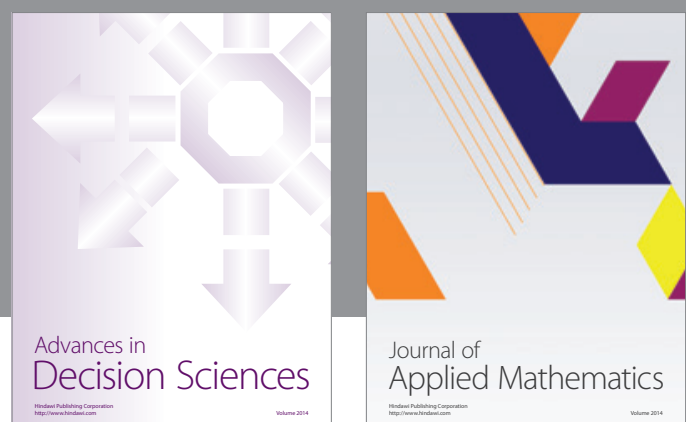

Journal of

Applied Mathematics
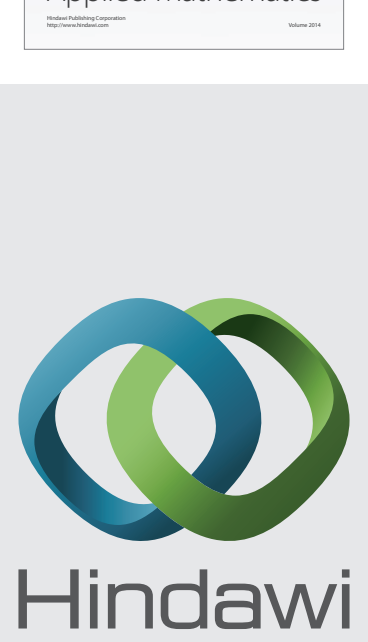

Submit your manuscripts at http://www.hindawi.com
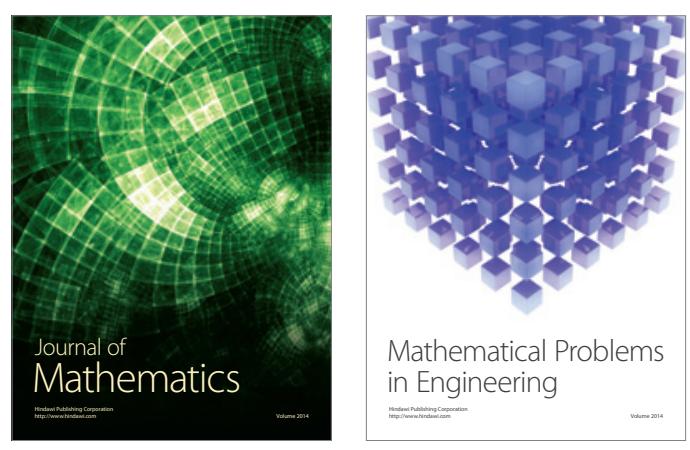

Mathematical Problems in Engineering
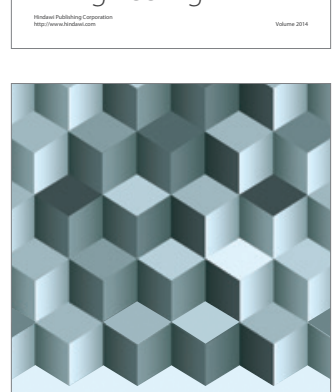

Journal of

Function Spaces
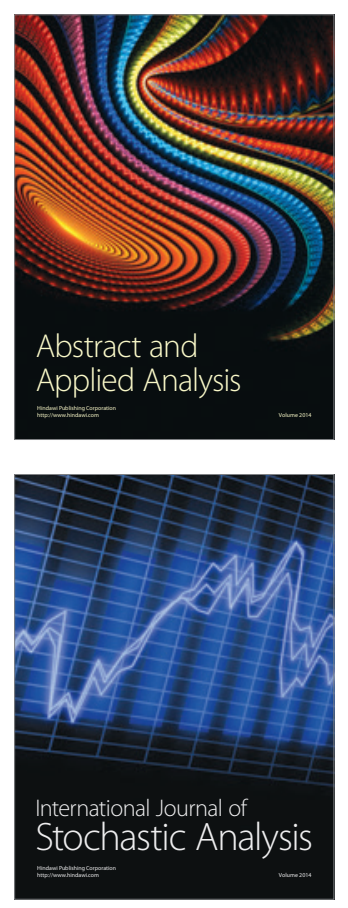

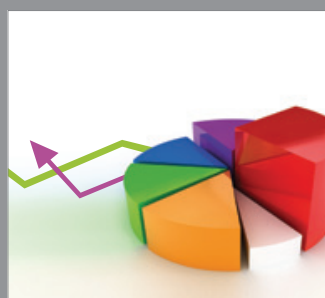

ournal of

Probability and Statistics

Promensencen
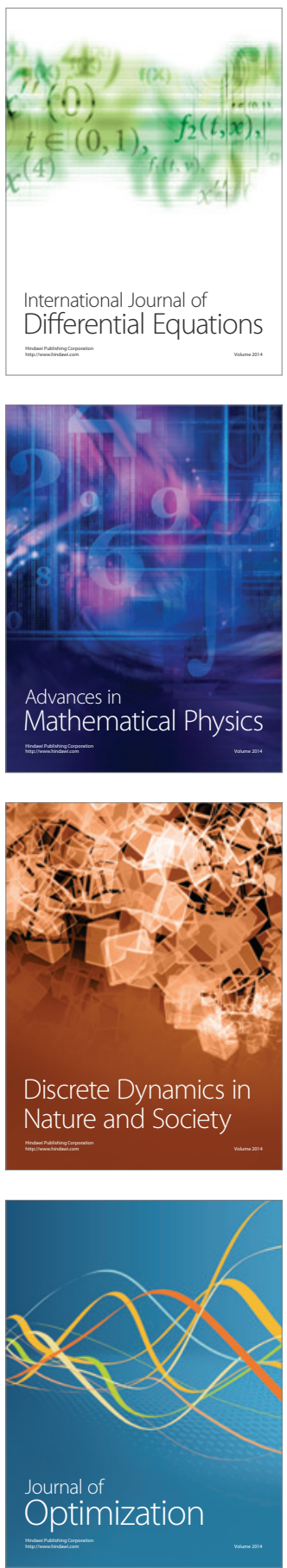